\title{
Longitudinal leading-twist distribution amplitude of the $J / \psi$ meson within the background field theory
}

\author{
Hai-Bing Fu and Long Zeng \\ Department of Physics, Guizhou Minzu University, Guiyang 550025, People's Republic of China \\ Wei Cheng and Xing-Gang Wu* \\ Department of Physics, Chongqing University, Chongqing 401331, People's Republic of China \\ Tao Zhong \\ Physics Department, Henan Normal University, Xinxiang 453007, People's Republic of China
}

(Received 22 January 2018; published 24 April 2018)

\begin{abstract}
We make a detailed study on the $J / \psi$ meson longitudinal leading-twist distribution amplitude $\phi_{2 ; J / \psi}^{\|}$by using the QCD sum rules within the background field theory. By keeping all the nonperturbative condensates up to dimension 6, we obtain accurate QCD sum rules for the moments $\left\langle\xi_{n ; J / \psi}^{\|}\right\rangle$. The first three ones are $\left\langle\xi_{2 ; J / \psi}\right\rangle=0.083(12),\left\langle\xi_{4 ; J / \psi}\right\rangle=0.015(5)$, and $\left\langle\xi_{6 ; J / \psi}^{\|}\right\rangle=0.003(2)$, respectively. Those values indicate a single peaked behavior for $\phi_{2 ; J / \psi}^{\|}$. As an application, we adopt the QCD light-cone sum rules to calculate the $B_{c}$ meson semileptonic decay $B_{c}^{+} \rightarrow J / \psi \ell^{+} \nu_{\ell}$. We obtain $\Gamma\left(B_{c}^{+} \rightarrow J / \psi \ell^{+} \nu_{\ell}\right)=$ $\left(89.67_{-19.06}^{+24.76}\right) \times 10^{-15} \mathrm{GeV}$ and $\mathfrak{R}\left(J / \psi \ell^{+} \nu_{\ell}\right)=0.217_{-0.057}^{+0.069}$, which agree with both the extrapolated next-to-leading order $\mathrm{pQCD}$ prediction and the new CDF measurement within errors.
\end{abstract}

DOI: $10.1103 /$ PhysRevD.97.074025

\section{INTRODUCTION}

The $B_{c}^{+}$meson has been discovered by the Collider Detector at Fermilab (CDF) Collaboration via the semileptonic decay channel $B_{c}^{+} \rightarrow J / \psi \ell^{+} \nu_{\ell}$ [1]. At the same time, they also measured the ratio of the production cross sections times branching fractions of the $B_{c}^{+}$meson in the decay mode $B_{c}^{+} \rightarrow J / \psi \ell^{+} \nu_{\ell}$ to the $B^{+}$meson in the decay mode $B^{+} \rightarrow J / \psi K^{+}$, i.e.,

$$
\begin{gathered}
\Re\left(J / \psi \ell^{+} \nu_{\ell}\right)=\frac{\sigma\left(B_{c}^{+}\right) \mathcal{B}\left(B_{c}^{+} \rightarrow J / \psi \ell^{+} \nu_{\ell}\right)}{\sigma\left(B^{+}\right) \mathcal{B}\left(B^{+} \rightarrow J / \psi K^{+}\right)} \\
=0.132_{-0.037}^{+0.041}(\mathrm{st}) \pm 0.031(\mathrm{sy})_{-0.020}^{+0.032}(\text { lf })
\end{gathered}
$$

where the symbols "st", "sy," and "lf" stand for the statistical error, the systematic error, and the error of the $B_{c}$ meson lifetime, respectively. In year 2016, the CDF Collaboration updates the value of $\Re\left(J / \psi \ell^{+} \nu_{\ell}\right)$ by using

\footnotetext{
*Corresponding author. wuxg@cqu.edu.cn

Published by the American Physical Society under the terms of the Creative Commons Attribution 4.0 International license. Further distribution of this work must maintain attribution to the author(s) and the published article's title, journal citation, and DOI. Funded by SCOAP ${ }^{3}$.
}

the CDF Run II data with an integrated luminosity $8.7 \mathrm{fb}^{-1}$, i.e., $\Re\left(J / \psi \ell^{+} \nu_{\ell}\right)=0.211 \pm 0.012(\mathrm{st})_{-0.020}^{+0.021}$ (sy) [2].

In the literature, the decay width for the semileptonic decay $B_{c}^{+} \rightarrow J / \psi \ell^{+} \nu_{\ell}$ has been calculated under various frameworks, such as the constituent quark model (CQM) $[3,4]$, the Bethe-Salpeter (BS) equation [5], the relativistic potential model (PM) [6], the perturbative QCD (pQCD) theory [7], the QCD sum rules (QCD SR) [8,9], and the QCD light-cone sum rules (QCD LCSR) [10,11]. In those predictions, the decay width is always small, leading to a large discrepancy between the experiment and theoretical predictions on $\Re\left(J / \psi \ell^{+} \nu_{\ell}\right)$.

Many effects have been tried to solve this discrepancy. In 2013, a next-to-leading order (NLO) pQCD calculation gives $\quad \Gamma\left(B_{c}^{+} \rightarrow J / \psi \ell^{+} \nu_{\ell}\right)=\left(97.30_{-20.33}^{+36.22}\right) \times 10^{-15} \mathrm{GeV}$ [12], whose accuracy has lately been improved by applying the principle of maximum conformality (PMC) [13-16] scale-setting approach such that there is no renormalization scale independence in the decay width [17], which gives $\Gamma\left(B_{c}^{+} \rightarrow J / \psi \ell^{+} \nu_{\ell}\right)=\left(106.31_{-14.01}^{+18.59}\right) \times 10^{-15} \mathrm{GeV}$. Those pQCD predictions lead to a larger ratio $\Re\left(J / \psi \ell^{+} \nu_{\ell}\right)$ in agreement with the CDF Run II data. However, the pQCD calculation for the $B_{c} \rightarrow J / \psi$ transition form factor (TFF) is only reliable in large recoil region $q^{2} \sim 0$, which should be extended to the whole $q^{2}$ region via a model-dependent extrapolation. This introduces extra model dependence into 
pQCD predictions; thus predictions from other approaches are helpful for a cross-check.

On the other hand, the TFFs under the QCD SR and LCSR approaches are reliable for both the low and intermediate $q^{2}$ region. To compare with the pQCD predictions, more reliable predictions could be expected by applying those two approaches. However, previous QCD SR or QCD LCSR predictions on those TFFs are quite small [8-11], leading to a smaller $\Re\left(J / \psi \ell^{+} \nu_{\ell}\right)$ well below the measured value. It is thus helpful to know whether the QCD SR or QCD LCSR prediction can be improved by carefully reconsidering its key components such as the TFFs and the light-cone distribution amplitudes (LCDAs) of the $J / \psi$ meson.

The QCD LCSR is based on the operator product expansion near the light cone $x^{2} \rightsquigarrow 0$, which parametrizes all the nonperturbative dynamics into the LCDAs. Those LCDAs are nonperturbative but universal, which is a key component to exclusive processes. Up to twist- 4 accuracy, there are totally fifteen LCDAs for the vector meson. For the light vector meson such $\rho$ or $K^{*}$, it is helpful to distribute the LCDA contributions in the $B \rightarrow$ light vector meson TFFs by using the parameter $\delta_{\rho} \sim m_{\rho} / m_{B} \sim 0.16$ or $\delta_{K^{*}} \sim m_{K}^{*} / m_{B} \sim 0.17$ [18-20]. We can define a similar parameter $\delta_{J / \psi}$ for the $J / \psi$ meson, whose value is much larger than that of the light vector, i.e., $\delta_{J / \psi} \sim 0.50>\delta_{\rho}$ or $\delta_{K^{*}}$. So we do not use the parameter $\delta_{J / \psi}$ to classify the $J / \psi$ meson LCDAs.

As a tricky point of the LCSR approach, one can highlight the wanted LCDA contributions and suppress the unwanted LCDA contributions to the LCSR by choosing a proper chiral correlator [21-23]. For example, by using a left-handed chiral correlator, we have calculated the LCSRs for the $B \rightarrow \rho$ TFFs in Refs. [24,25]. By further replacing the $\rho$-meson LCDAs with those of the $J / \psi$ meson, we obtain the LCSRs for the $B_{c} \rightarrow J / \psi$ TFFs. It is found that the resultant LCSRs for the $B_{c} \rightarrow J / \psi$ TFFs highlight the contributions from the chiral-even $J / \psi$ meson LCDAs $\phi_{2 ; J / \psi}^{\|}, \phi_{3 ; J / \psi}^{\perp}, \psi \stackrel{1}{\perp ; J / \psi}_{3}^{\perp}, \Phi_{3 ; J / \psi}^{\|}, \tilde{\Phi}_{3 ; J / \psi}^{\|}, \phi_{4 ; J / \psi}^{\|}$, and $\psi_{4 ; J / \psi}^{\|}$, respectively, while, the chiral-odd LCDAs $\phi_{2 ; J / \psi}^{\perp}$, $\phi_{3 ; \rho}^{\|}, \psi_{3 ; J / \psi}^{\|}, \Phi_{3 ; J / \psi}^{\perp}, \phi_{4 ; J / \psi}^{\perp}, \psi_{4 ; J / \psi}^{\perp}, \Psi_{4 ; J / \psi}^{\perp}$, and $\tilde{\Psi}_{4 ; J / \psi}^{\perp}$ give zero contributions to the TFFs. Among the nonzero chiraleven LCDAs, the LCDAs $\phi_{2 ; J / \psi}^{\|}, \phi_{3 ; J / \psi}^{\perp}$, and $\psi_{3 ; J / \psi}^{\perp}$ provide dominant contributions to the TFFs, and the contributions from $\phi_{4 ; J / \psi}^{\|}, \psi_{4 ; J / \psi}^{\|}, \Phi_{3 ; J / \psi}^{\|}$, and $\tilde{\Phi}_{3 ; J / \psi}^{\|}$are negligible which are similar to the case of the $\rho$ meson. Furthermore, the LCDAs $\psi \stackrel{\perp}{\stackrel{\perp}{3} J / \psi}$ and $\phi_{3 ; J / \psi}^{\perp}$ can be related to the leading-twist LCDA $\phi_{2 ; J / \psi}^{\|}$under the Wandzura-Wilczek approximation [26]. Thus, by using a left-handed chiral correlator, our main task is to determine a precise $\phi_{2 ; J / \psi^{*}}^{\|}$.

Several models for the twist-2 LCDA $\phi_{2 ; J / \psi}^{\|}$have been suggested in the literature. For example, Bondar and
Chernyak [27], Bodwin et al. [28], and Sun et al. [29] suggested three different models to resolve the disagreement between the experimental observations and the theoretical predictions on the production cross section of the process $e^{+} e^{-} \rightarrow J / \psi+\eta_{c}$.

Generally, the twist-2 LCDA $\phi_{2 ; J / \psi}^{\|}$at the scale $\mu$ can be expanded in a Gegenbauer polynomial as [30]

$$
\phi_{2 ; J / \psi}^{\|}(x, \mu)=6 x \bar{x}\left[1+\sum_{n=1}^{\infty} a_{n ; J / \psi}^{\|}(\mu) C_{n}^{3 / 2}(\xi)\right],
$$

where $a_{n ; J / \psi}^{\|}(\mu)$ stands for the $n$ th-order Gegenbauer moment. $\bar{x}=1-x$ and $\xi=x-\bar{x}$. When the scale $\mu$ is large enough, the twist-2 LCDA $\phi_{2 ; J / \psi}^{\|}(x, \mu)$ tends to the well-known asymptotic form $6 x \bar{x}$ [31].

In the paper, we study the properties of $\phi_{2 ; J / \psi}^{\|}$via studying its moments by using the Shifman-VainshteinZakharov (SVZ) sum rules [32] under the background field theory (BFT). The SVZ sum rules relate the hadronic parameters, such as the meson masses and strong coupling constants, the baryon magnetic moments, etc., to a few nonperturbative gluon and quark condensates. Those condensates are universal, and once we have determined their values by comparing with the known observables, they can be applied to all observables involving them. The SVZ sum rules approach has been applied, with remarkable success, for a large variety of properties of the low-lying hadronic states. The BFT provides a self-consistent description on those vacuum condensates and provides a systematic way to achieve the goal of the SVZ sum rules $[33,34]$. The SVZ sum rules for the $J / \psi$ meson LCDAs are much more involved than the light vector LCDAs, since we have to take the charm-quark mass effect into consideration. Fortunately, Ref. [35] has given the quark propagator and vertex operator $(z \cdot \stackrel{\leftrightarrow}{D})^{n}$ with full mass dependence within the framework of BFT. Thus one can derive precise SVZ sum rules for the moments of $\phi_{2 ; J / \psi}^{\|}$with full mass dependence, as is the purpose of the paper.

The remaining parts of the paper are organized as follows. In Sec. II, we present the SVZ sum rules for the moments of $\phi_{2 ; J / \psi}^{\|}$. Properties of the resultant $\phi_{2 ; J / \psi}^{\|}$, together with its application for the semileptonic decay $B_{c}^{+} \rightarrow J / \psi \ell^{+} \nu_{\ell}$, are discussed in Sec. III. The final section is reserved for a summary.

\section{CALCULATION TECHNOLOGY}

\section{A. SVZ sum rules for the moments of $\phi_{2 ; J / \psi}^{\|}$}

The QCD Lagrangian within the framework of BFT can be obtained from the conventional QCD Lagrangian by replacing the gluon field $\mathcal{A}_{\mu}^{A}(x)$ and quark field $\psi(x)$ to the following ones: 


$$
\begin{gathered}
\mathcal{A}_{\mu}^{A}(x) \rightarrow \mathcal{A}_{\mu}^{A}(x)+\phi_{\mu}^{A}(x), \\
\psi(x) \rightarrow \psi(x)+\eta(x) .
\end{gathered}
$$

Here $\mathcal{A}_{\mu}^{A}(x)$ with $A=(1, \ldots, 8)$ and $\psi(x)$ are gluon and quark background fields. $\phi_{\mu}^{A}(x)$ and $\eta(x)$ are gluon and quark quantum fields, i.e., the quantum fluctuation on the background fields. The QCD Lagrangian within the BFT is given by Ref. [34]. The background fields satisfy the equations of motion

$$
(i D-m) \psi(x)=0
$$

and

$$
\tilde{D}_{\mu}^{A B} G^{B \nu \mu}(x)=g_{s} \bar{\psi}(x) \gamma^{\nu} T^{A} \psi(x),
$$

where $D_{\mu}=\partial_{\mu}-i g_{s} T^{A} \mathcal{A}_{\mu}^{A}(x)$ and $\tilde{D}_{\mu}^{A B}=\delta^{A B}-g_{s} f^{A B C} \times$ $\mathcal{A}_{\mu}^{C}(x)$ are fundamental and adjoint representations of the gauge covariant derivative, respectively.

The physical observables should be gauge independent; one may take different gauges for the quantum fluctuations and the background fields such as to make the sum rules calculation relatively simpler. Practically, we adopt the background gauge, $\tilde{D}_{\mu}^{A B} \phi^{B \mu}(x)=0$, for the gluon quantum field [33,36,37], and the Schwinger gauge or the fixedpoint gauge, $x^{\mu} \mathcal{A}_{\mu}^{A}(x)=0$, for the background field [38]. Using those inputs, the quark propagator $S_{F}(x, 0)$ and the vertex operators $\Gamma(z \cdot \stackrel{\leftrightarrow}{D})^{n}$ are ready to be derived, whose explicit expressions up to dimension-six operators can be found in Ref. [35].

The twist-2 LCDA $\phi_{2 ; J / \psi}^{\|}(x, \mu)$ is defined via the following equation,

$$
\begin{aligned}
& \left\langle 0\left|\bar{Q}_{1}(z) \not d Q_{2}(-z)\right| J / \psi\right\rangle \\
& \quad=i(z \cdot q) f_{J / \psi}^{\|} \int_{0}^{1} d x e^{i \xi(z \cdot q)} \phi_{2 ; J / \psi}^{\|}(x, \mu),
\end{aligned}
$$

where $\xi=2 x-1$ and $f_{J / \psi}^{\|}$is the $J / \psi$ meson decay constant. It leads to

$$
\begin{aligned}
& \left\langle 0\left|\bar{Q}(0) \not(i z \cdot \stackrel{\leftrightarrow}{D})^{n} Q(0)\right| J / \psi\right\rangle \\
& \quad=\left(e^{(\lambda) *} \cdot z\right)(q \cdot z)^{n} m_{J / \psi} f_{J / \psi}^{\|}\left\langle\xi_{n, J / \psi}^{\|}\right\rangle,
\end{aligned}
$$

where $q$ and $e^{(\lambda)}$ are the momentum and polarization vector of $J / \psi$ meson, $(z \cdot \stackrel{\leftrightarrow}{D})^{n}=(z \cdot \vec{D}-z \cdot \overleftarrow{D})^{n}$. The $n$ th-order moment $\left\langle\xi_{n ; J / \psi}^{\|}\right\rangle$at the scale $\mu$ is defined as

$$
\left\langle\xi_{n ; J / \psi}^{\|}\right\rangle=\int_{0}^{1} d x \xi^{n} \phi_{2 ; J / \psi}^{\|}(x, \mu) .
$$

As a special case, the 0th moment satisfies the normalization condition

$$
\left\langle\xi_{0 ; J / \psi}^{\|}\right\rangle=\int_{0}^{1} d x \phi_{2 ; J / \psi}^{\|}(x, \mu)=1
$$

To derive the SVZ sum rules for the moments $\left\langle\xi_{n ; J / \psi}\right\rangle$, we introduce the following correlator,

$$
\begin{aligned}
\Pi_{J / \psi}^{(n, 0)}(z, q) & =i \int d^{4} x e^{i q \cdot x}\left\langle 0\left|T\left\{J_{n}(x) J_{0}^{\dagger}(0)\right\}\right| 0\right\rangle \\
& =(z \cdot q)^{n+2} I^{(n, 0)}\left(q^{2}\right),
\end{aligned}
$$

where $J_{n}(x)=\bar{Q}(x) \not(i z \cdot \stackrel{\leftrightarrow}{D})^{n} Q(x)$ and $z^{2}=0$. For the present $c \bar{c}$ system, only even moments are nonzero, $n=(0,2,4, \ldots)$.

The correlator (12) is an analytic $q^{2}$ function.

In the physical region $\left(q^{2}>0\right)$, the hadronic content of the correlator can be quantified by inserting a complete set of the intermediate hadronic states into the matrix element with the help of the unitarity relation. By further singling out the ground state and introducing a compact notation for the rest of contributions including excited vector mesons and continuum states, we obtain

$$
\begin{aligned}
\frac{1}{\pi} \operatorname{Im} I_{\text {had }}^{(n, 0)}\left(q^{2}\right)= & \delta\left(q^{2}-m_{J / \psi}^{2}\right) f_{J / \psi}^{\| 2}\left\langle\xi_{n ; J / \psi}^{\|}\right\rangle \\
& +\frac{3}{4 \pi^{2}(n+1)(n+3)} \theta\left(q^{2}-s_{J / \psi}\right),
\end{aligned}
$$

where the quark-hadron duality has been adopted and the symbol $s_{J / \psi}$ stands for the continuum threshold for the lowest continuum state.

In deep Euclidean region $q^{2}<0$, one can apply the operator product expansion for the correlator (12), and the coefficients before the operators are perturbatively calculable. As a combination of the correlator within the different $q^{2}$ region, the sum rules for $\left\langle\xi_{n ; J / \psi}^{\|}\right\rangle$can be derived by using the dispersion relation. As a final step, the Borel transformation is always applied such that to suppress the contributions from excited and continuum states and those from high dimensional operators.

Following standard SVZ sum rules procedures [32,39], the final sum rules read 


$$
\begin{aligned}
& \left\langle\xi_{n ; J / \psi}^{\|}\right\rangle=\frac{e^{m_{J / \Psi}^{2} / M^{2}}}{f_{J / \Psi}^{\| 2}}\left\{\frac{3}{8 \pi^{2}(n+1)(n+3)}\left(1+\frac{\alpha_{s}}{\pi} A_{n}^{\prime}\right) \int_{t_{\min }}^{s_{J / \psi}} d s e^{-s / M^{2}}\left[v^{n+1} \frac{2(n+1) m_{c}^{2}+s}{s}-(v \rightarrow-v)\right]+\frac{\left\langle\alpha_{s} G^{2}\right\rangle}{6 \pi M^{2}}\right. \\
& \times \int_{0}^{1} d x e^{-\frac{m_{c}^{2}}{x \bar{x} M^{2}}} \frac{\xi^{n-2}}{x^{2} \bar{x}^{2}}\left[n(n-1) x^{3} \bar{x}^{3}+\frac{\xi^{2}}{2}\left(1-\frac{m_{c}^{2}\left(x^{3}+\bar{x}^{3}\right)}{x^{3} \bar{x}^{3} M^{2}}\right)\right]+\frac{\left\langle g_{s}^{3} f G^{3}\right\rangle}{16 \pi^{2} M^{4}} \int_{0}^{1} d x e^{-\frac{m_{c}^{2}}{x \bar{x} M^{2}}} \frac{\xi^{n-2}}{2} \\
& \times\left\{\left[-\xi^{2}\left(\frac{69+2 n(11+64 x \bar{x})}{72 x \bar{x}}+\frac{45(1-3 x \bar{x})}{8 x^{2} \bar{x}^{2}}\right)-\frac{n(n-1)}{9}[16+(n-31) x \bar{x}]\right]+\frac{1}{3 M^{2}}\left[\xi ^ { 2 } \left(\frac{m_{c}^{2}(1+2 x \bar{x})}{12 x^{2} \bar{x}^{2}}\right.\right.\right. \\
& \left.\left.\left.\left.-\frac{8 n m_{c}^{2}}{3 x \bar{x}}-\frac{3 m_{c}^{2}\left(x^{4}+\bar{x}^{4}\right)}{4 x^{3} \bar{x}^{3}}\right)+\xi \frac{11 n m_{c}^{2}\left(x^{3}-\bar{x}^{3}\right)}{6 x^{2} \bar{x}^{2}}-\frac{n(n-1) m_{c}^{2}}{3}\right]+\xi^{2} \frac{m_{c}^{2}\left(x^{5}+\bar{x}^{5}\right)}{30 M^{4} x^{4} \bar{x}^{4}}\right\}\right\} \text {. }
\end{aligned}
$$

As an estimation of the NLO coefficients $A_{n}^{\prime}$, we adopt the ones without quark mass effect. The first four ones are [40], $A_{0}^{\prime}=1, A_{2}^{\prime}=5 / 3, A_{4}^{\prime}=59 / 27$, and $A_{6}^{\prime}=353 / 135$, respectively. By using the moments $\left\langle\xi_{n ; J / \psi}^{\|}\right\rangle$, we can obtain the Gegenbauer moments $a_{n ; J / \psi}^{\|}$up to $n=6$ by using the following relations, i.e.,

$$
\begin{aligned}
& \left\langle\xi_{2 ; J / \psi}^{\|}\right\rangle=\frac{1}{5}+\frac{12}{35} a_{2 ; J / \psi}^{\|}, \\
& \left\langle\xi_{4 ; J / \psi}^{\|}\right\rangle=\frac{3}{35}+\frac{8}{35} a_{2 ; J / \psi}^{\|}+\frac{8}{77} a_{4 ; J / \psi}^{\|}, \\
& \left\langle\xi_{6 ; J / \psi}^{\|}\right\rangle=\frac{1}{21}+\frac{12}{77} a_{2 ; J / \psi}^{\|}+\frac{120}{1001} a_{4 ; J / \psi}^{\|}+\frac{64}{2145} a_{6 ; J / \psi}^{\|} .
\end{aligned}
$$

$$
\cdots
$$

Those relations are obtained by applying the Gegenbauer polynomial expansion of $\phi_{2 ; J / \psi}^{\|}$[Eq. (3)] into Eq. (10).

\section{B. The semileptonic decay for $B_{c}^{+} \rightarrow J / \psi \ell^{+} \nu \ell$}

The differential decay width for the semileptonic decay $B_{c}^{+}(P) \rightarrow J / \psi(p) \ell^{+} \nu_{\ell}$ over the momentum transfer $q^{2}$ can be formulated as

$$
\begin{gathered}
\frac{\mathrm{d} \Gamma_{L}\left(B_{c}^{+} \rightarrow J / \psi \ell^{+} \nu_{\ell}\right)}{\mathrm{d} q^{2}}=\left(\frac{q^{2}-m_{\ell}^{2}}{q^{2}}\right)^{2} \frac{\sqrt{\lambda\left(q^{2}\right)} G_{F}^{2}\left|V_{\mathrm{cb}}\right|^{2}}{384 m_{B_{c}^{+}}^{3} \pi^{3}}\left[\frac{3 m_{\ell}^{2}}{q^{2}} \lambda\left(q^{2}\right) A_{0}^{2}\left(q^{2}\right)+\left(m_{\ell}^{2}+2 q^{2}\right)\left|h_{0}\left(q^{2}\right)\right|^{2}\right], \\
\frac{\mathrm{d} \Gamma_{T}\left(B_{c}^{+} \rightarrow J / \psi \ell^{+} \nu_{\ell}\right)}{\mathrm{d} q^{2}}=\left(\frac{q^{2}-m_{\ell}^{2}}{q^{2}}\right)^{2} \frac{\sqrt{\lambda\left(q^{2}\right)} G_{F}^{2}\left|V_{\mathrm{cb}}\right|^{2}}{384 m_{B_{c}^{+}}^{3} \pi^{3}}\left(m_{\ell}^{2}+2 q^{2}\right)\left[\left|h_{+}\left(q^{2}\right)\right|^{2}+\left|h_{-}\left(q^{2}\right)\right|^{2}\right],
\end{gathered}
$$

where $q=P-p$ is the momentum transfer of this process, the Fermi constant $G_{F}=1.16638 \times 10^{-5}$, and the phasespace factor $\lambda\left(q^{2}\right)=\left(m_{B_{c}^{+}}^{2}+m_{J / \psi}^{2}-q^{2}\right)^{2}-4 m_{B_{c}^{+}}^{2} m_{J / \psi}^{2}$. Here we have separated the decay width into longitudinal and transverse ones as $\Gamma=\Gamma_{L}+\Gamma_{T}$. In this paper, the lepton is taken as the light ones, e.g., $\ell=e$ or $\mu$, respectively. Thus the TFF $A_{0}\left(q^{2}\right)$ contributes 0 to the decay width due to the chiral suppression.

The longitudinal and transverse helicity amplitudes for the decay widths $\Gamma_{L}$ and $\Gamma_{T}$ are

$$
\begin{aligned}
h_{ \pm}\left(q^{2}\right)= & \frac{\sqrt{\lambda\left(q^{2}\right)}}{m_{B_{c}^{+}}+m_{J / \psi}}\left[V\left(q^{2}\right) \mp \frac{\left(m_{B_{c}^{+}}+m_{J / \psi}\right)^{2}}{\sqrt{\lambda\left(q^{2}\right)}}\right. \\
& \left.\times A_{1}\left(q^{2}\right)\right]
\end{aligned}
$$

$$
\begin{aligned}
h_{0}\left(q^{2}\right)= & \frac{1}{2 m_{J / \psi} \sqrt{q^{2}}}\left[-\frac{\lambda\left(q^{2}\right)}{m_{B_{c}^{+}}+m_{J / \psi}} A_{2}\left(q^{2}\right)\right. \\
& \left.+\left(m_{B_{c}^{+}}+m_{J / \psi}\right)\left(m_{B_{c}^{+}}^{2}-m_{J / \psi}^{2}-q^{2}\right) A_{1}\left(q^{2}\right)\right] .
\end{aligned}
$$

The four $B_{c} \rightarrow J / \psi$ TFFs $V\left(q^{2}\right), A_{0}\left(q^{2}\right), A_{1}\left(q^{2}\right)$, and $A_{2}\left(q^{2}\right)$, as mentioned in the introduction, can be read from Refs. [24,25], which are either directly or indirectly related to the twist-2 LCDA $\phi_{2 ; J / \psi}^{\|}$.

\section{NUMERICAL RESULTS}

We adopt the following parameters to do the numerical calculation. Two nonperturbative gluon condensates are 


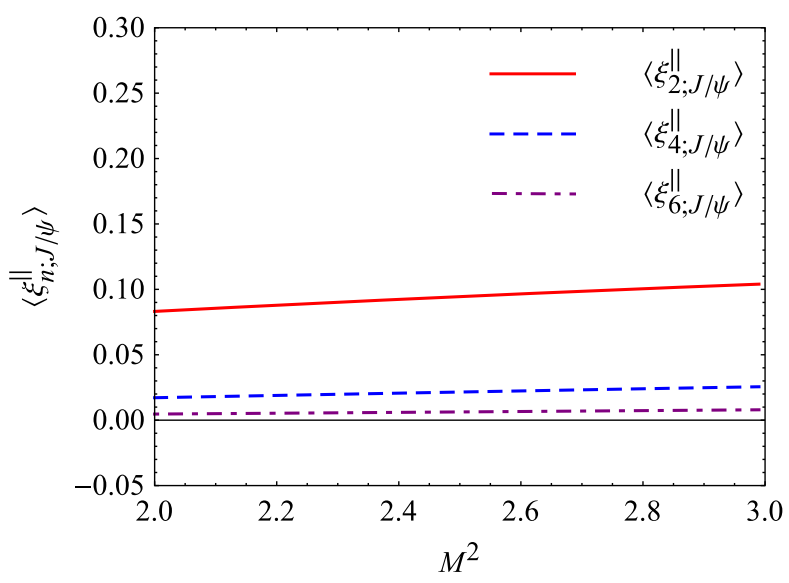

FIG. 1. The first three moments $\left\langle\xi_{n ; J / \psi}^{\|}\right\rangle(n=2,4,6)$ versus the Borel parameter $M^{2}$. All input parameters are taken as their central values.

taken as $\left\langle\alpha_{s} G^{2}\right\rangle=0.038(11) \mathrm{GeV}^{4} \quad$ and $\quad\left\langle g_{s}^{3} f G^{3}\right\rangle=$ $0.013(7) \mathrm{GeV}^{6}[35,39]$. The current charm-quark mass, $\bar{m}_{c}\left(\bar{m}_{c}\right)=1.275 \pm 0.025 \mathrm{GeV}$, the masses of $B_{c}^{+}$and $J / \psi$ mesons are $m_{B_{c}^{+}}=6.274 \mathrm{GeV}$ and $m_{J / \psi}=3.097 \mathrm{GeV}$ from the Particla data group (PDG) [41]. The $J / \psi$ decay constant can be related to its leptonic decay width $\Gamma_{J / \psi \rightarrow e^{+} e^{-}}$via the relation [42],

$$
f_{J / \psi}^{\| 2}=\frac{3}{4 \pi \alpha^{2} c_{J / \psi}} m_{J / \psi} \Gamma_{J / \psi \rightarrow e^{+} e^{-}}
$$

where $\alpha=1 / 137$ and $c_{J / \psi}=4 / 9$. Taking the PDG averaged value, $\Gamma_{J / \psi \rightarrow e^{+} e^{-}}=5.547 \pm 0.14 \mathrm{KeV} \quad[41]$, we obtain $f_{J / \psi}^{\|}=416.2 \pm 5.3 \mathrm{MeV}$.

\section{A. The $J / \psi$ meson leading-twist LCDA $\phi_{2 ; J / \psi}^{\|}(x, \mu)$}

We set the continuum threshold $s_{J / \psi}$ for the moments $\left\langle\xi_{n: J / \psi}^{\|}\right\rangle$as the value around the squared mass of the $J / \psi$ meson's first excited state. The structure of the excited $J / \psi$ meson state is not yet clear; as suggested by Braguta et al. [43], we set the value of $s_{J / \psi}$ to infinity.

To determine the allowable range of $M^{2}$, i.e., the Borel window, for the sum rules of the moments $\left\langle\xi_{n ; J / \psi}\right\rangle$, we adopt three usually used criteria: (I) The continuum contributions are less than $40 \%$ of the total dispersion relation; (II) the contributions from the dimension-six condensates do not exceed 10\%; (III) the flatness of the moments versus $M^{2}$, since the moments should be independent to the Borel parameter $M^{2}$ when all $1 / M^{2}$ terms have been summed up. For definiteness, we require the variations of the moments within the Borel window be less than $20 \%$. This could be treated as the residual $1 / M^{2}$ dependence due to the fact that we only know the series up to $1 / M^{4}$. Figure 1 shows how the moments $\left\langle\xi_{n ; J / \psi}^{\|}\right\rangle$vary with $M^{2}$. The determined Borel window is $M^{2} \in$ $[2,3] \mathrm{GeV}^{2}$; within this range the moments $\left\langle\xi_{4 ; J / \psi}^{\|}\right\rangle$and $\left\langle\xi_{6 ; J / \psi}^{\|}\right\rangle$are almost flat, and the moment $\left\langle\xi_{2 ; J / \psi}^{\|}\right\rangle$changes by $\sim \pm 10 \%$.

We present the moments $\left\langle\xi_{(2,4,6) ; J / \psi}^{\|}\right\rangle$at the scale $\mu=M$ in Table I, where the perturbative contributions are calculated up to NLO level and the nonperturbative contributions are up to dimension-six condensates. The errors are squared averages of the uncertainties from the Borel parameter, the nonperturbative gluon condensates, and the $c$-quark mass. Table I shows that the dominant contribution is from the LO terms, which provide $\sim 95 \%$ contribution to $\left\langle\xi_{2 ; J / \psi}^{\|}\right\rangle, \sim 91 \%$ to $\left\langle\xi_{4 ; J / \psi}^{\|}\right\rangle$, and $\sim 94 \%$ to $\left\langle\xi_{6 ; J / \psi}^{\|}\right\rangle$, respectively. The NLO terms provide $\sim 6.0 \%$ contribution to $\left\langle\xi_{2 ; J / \psi}^{\|}\right\rangle, \sim 7 \%$ contribution to $\left\langle\xi_{4 ; J / \psi}^{\|}\right\rangle$, and $\sim 10 \%$ contribution to $\left\langle\xi_{6 ; J / \psi}^{\|}\right\rangle$. Contributions of the high dimensional condensates are small, and the condensates do not follow the usual power counting of $1 / M^{2}$ suppression. Contribution from the dimension-six condensate has the same importance as that of the dimension-four condensate; thus both of them should be treated on an equal footing.

Using the relations among the Gegenbauer moments $a_{n ; J / \psi}^{\|}$and the moments $\left\langle\xi_{n ; J / \psi}^{\|}\right\rangle$, we can get $a_{n ; J / \psi}^{\|}$at the same scale. The Gegenbauer moments $a_{n ; J / \psi}^{\|}$at any other scale can be obtained via the QCD evolution. At the NLO accuracy, we have [44-46]

$$
\begin{aligned}
a_{n ; J / \psi}^{\|}(\mu)= & a_{n ; J / \psi}^{\|}\left(\mu_{0}\right) E_{n ; J / \psi}^{\mathrm{NLO}} \\
& +\frac{\alpha_{s}(\mu)}{4 \pi} \sum_{k=0}^{n-2} a_{k ; J / \psi}\left(\mu_{0}\right) L^{\gamma_{k}^{(0)} /\left(2 \beta_{0}\right)} d_{n k}^{(1)} .
\end{aligned}
$$

TABLE I. The moments $\left\langle\xi_{(2,4,6) ; J / \psi}^{\|}\right\rangle$of the $J / \psi$ longitudinal twist-2 DA at the scale $\mu=M$. The contributions from the LO terms, the NLO terms, the dimension-four condensates, and the dimension-six condensates are presented separately. The errors are the squared average of all the mentioned error sources.

\begin{tabular}{lccccc}
\hline \hline & LO & NLO & Dimension 4 & Dimension 6 & Total \\
\hline$\left\langle\xi_{2 ; J / \psi}^{\|}\right\rangle$ & $0.0890(77)$ & $0.0056(5)$ & $0.0001(3)$ & $-0.0010(7)$ & $0.0937(108)$ \\
$\left\langle\xi_{4, J / \psi}^{\|}\right\rangle$ & $0.0195(32)$ & $0.0016(3)$ & $0.0007(5)$ & $-0.0004(2)$ & $0.0214(44)$ \\
$\left\langle\xi_{6 ; J / \psi}^{\|}\right\rangle$ & $0.0059(14)$ & $0.0006(1)$ & $0.0005(3)$ & $-0.0002(1)$ & $0.0063(17)$ \\
\hline \hline
\end{tabular}


TABLE II. The moments $\left\langle\xi_{n ; J / \psi}^{\|}\right\rangle$at the scale $\mu_{c}=\bar{m}_{c}\left(\bar{m}_{c}\right)$, where the errors are the squared average of all the mentioned error sources. The QCD SR prediction [47], the BT potential model [48], the Cornell potential model [49], and the NRQCD prediction [50] are also presented as a comparison.

\begin{tabular}{llll}
\hline \hline$\left\langle\xi_{n ; J / \psi}^{\|}\right\rangle$ & $n=2$ & $n=4$ & $n=6$ \\
\hline Our prediction & $0.083(12)$ & $0.015(5)$ & $0.003(2)$ \\
QCD SR [47] & $0.070(7)$ & $0.012(2)$ & $0.0031(8)$ \\
BT model [48] & 0.086 & 0.020 & 0.0066 \\
Cornell model [49] & 0.084 & 0.019 & 0.0066 \\
NRQCD [50] & $0.075(11)$ & $0.010(3)$ & $0.0017(7)$ \\
\hline \hline
\end{tabular}

Here $\mu_{0}$ is the initial scale, $\mu$ is the required scale, and

$$
\begin{aligned}
E_{n ; J / \psi}^{\mathrm{NLO}}= & L^{\gamma_{n}^{(0)} /\left(2 \beta_{0}\right)} \\
& \times\left\{1+\frac{\gamma_{n}^{(1)} \beta_{0}-\gamma_{n}^{(0)} \beta_{1}}{8 \pi \beta_{0}^{2}}\left[\alpha_{s}(\mu)-\alpha_{s}\left(\mu_{0}\right)\right]\right\},
\end{aligned}
$$

where $L=\alpha_{s}(\mu) / \alpha_{s}\left(\mu_{0}\right), \beta_{0}=11-2 n_{f} / 3, \beta_{1}=102-$ $38 n_{f} / 3$ with $n_{f}$ being the active flavor numbers; $\gamma_{n}^{(0)}$ and $\gamma_{n}^{(1)}$ are LO and NLO anomalous dimensions.

Taking the scale as $\mu_{c}=\bar{m}_{c}\left(\bar{m}_{c}\right)=1.275 \mathrm{GeV}$ and setting other parameters to be their central values, our predictions for the moments $\left\langle\xi_{n ; J / \psi}^{\|}\right\rangle$are listed in Table II. As a comparison, we also present the results derived within various approaches in Table II, i.e., the QCD SR [47], the Buchmuller-type (BT) potential model [48], the Cornell potential model [49], and the NRQCD [50]. Our results agree with other predictions within errors.

Using the relations (15), the first three Gegenbauer moments at the scale $\mu_{c}$ are

$$
\begin{aligned}
& a_{2 ; J / \psi}^{\|}=-0.340(34), \\
& a_{4 ; J / \psi}^{\|}=0.071(28), \\
& a_{6 ; J / \psi}^{\|}=0.002(1) .
\end{aligned}
$$

By substituting those Gegenbauer moments into Eq. (3), we show the $J / \psi$ meson longitudinal twist-2 LCDA in Fig. 2. As a comparison, we also present several other models in Fig. 2, i.e., the model suggested by Bondar and Chernyak (BC) [27],

$$
\phi_{\mathrm{BC}}(x)=c\left(v^{2}\right) x \bar{x}\left[\frac{x \bar{x}}{1-4 x \bar{x}\left(1-v^{2}\right)}\right],
$$

where $v^{2}=0.3$ and $c(0.3) \simeq 9.62$, the model constructed from the PM [28], and its asymptotic form $6 x \bar{x}$. Figure 2 shows that all LCDA models prefer a single-peaked

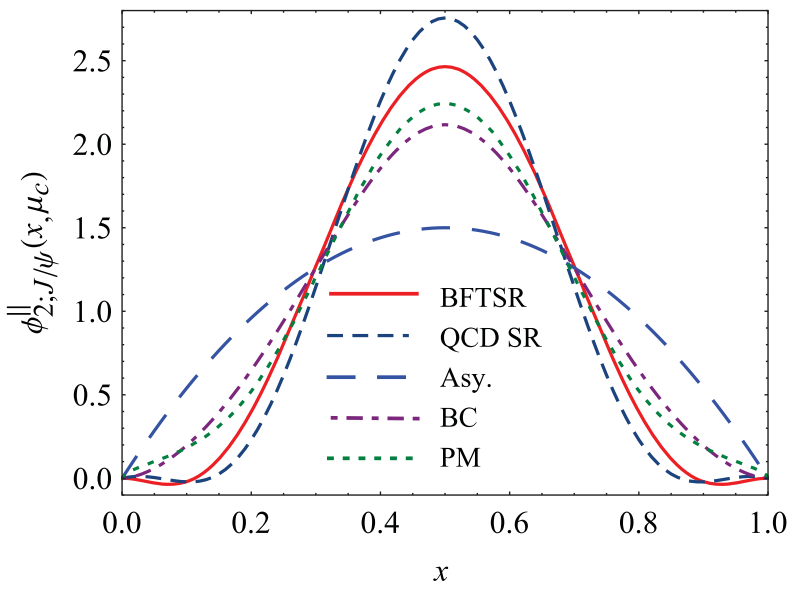

FIG. 2. The $J / \psi$ meson leading-twist LCDA $\phi_{2 ; J / \psi}^{\|}\left(x, \mu_{c}\right)$ predicted from the SVZ sum rules under the BFT (BFTSR). As a comparison, the asymptotic form, the QCD SR [47], BC model [27], and potential model [28] are also presented.

behavior; the BC and the PM LCDAs are close in shape. Our present LCDA has the sharper peak around $x \sim 0.5$ in agreement with the previous QCD SR prediciton [47], which has a stronger suppression around the end point $x \sim 0,1{ }^{1}$

\section{B. The $B_{c}^{+} \rightarrow J / \psi \ell^{+} \nu_{\ell}$ semileptonic decay}

One of the most important applications of the $J / \psi$ meson LCDAs is the $B_{c}$ meson semileptonic decay, $B_{c}^{+} \rightarrow J / \psi \ell^{+} \nu_{\ell}$. They are the key components of the $B_{c} \rightarrow J / \psi$ TFFs $A_{1}\left(q^{2}\right), A_{2}\left(q^{2}\right)$, and $V\left(q^{2}\right)$. By using a left-handed current $j_{B}^{\dagger}(x)=i \bar{b}(x)\left(1-\gamma_{5}\right) q_{2}(x)$ to do the LCSR calculation on the TFFs, one can suppress the contributions from other LCDAs and highlight the contributions from the longitudinal leading-twist LCDA $\phi_{2 ; J / \psi}^{\|}$, showing the properties of $\phi_{2 ; J / \psi}^{\|}$via a more transparent way. Thus the LCSRs derived by using the left-handed chiral correlator $[24,25]$ inversely provide good platforms for testing the behavior of $\phi_{2 ; J / \psi}^{\|}$.

To set the Borel window for the LCSRs of the $B_{c} \rightarrow J / \psi$ TFFs we adopt the following criteria:

(i) We require the continuum contribution to be less than $30 \%$ of the total LCSR.

(ii) We require all high-twist LCDAs contributions to be less than $15 \%$ of the total LCSR.

(iii) The derivatives of LCSRs for TFFs with respect to $\left(-1 / M^{2}\right)$ give three LCSRs for the $B_{c}$ meson mass $m_{B_{c}}$. We require the predicted $B_{c}$ meson mass to be fulfilled in comparing with the experiment one, e.g., $\left|m_{B_{c}}^{\text {th }}-m_{B_{c}}^{\exp }\right| / m_{B_{c}}^{\exp }$ less than $0.1 \%$.

\footnotetext{
${ }^{1}$ This behavior is helpful for suppressing the end-point singularity usually emerged in $B$ meson physics.
} 
TABLE III. The TFFs at the maximum recoil point $q^{2}=0$. The predictions from various approaches, such as the PMC prediction [17], the QCD SR prediction with a right-handed correlator [9], the three-point sum rule (3PSR) (with the Coloumb corrections being included) [8], and the QM [52], are presented as a comparison.

\begin{tabular}{lccc}
\hline \hline & $A_{1}(0)$ & $A_{2}(0)$ & $V(0)$ \\
\hline This work & $1.13_{-0.11}^{+0.13}$ & $1.20_{-0.12}^{+0.14}$ & $1.50_{-0.15}^{+0.17}$ \\
PMC [17] & $1.07(52)$ & $1.15(55)$ & $1.47(72)$ \\
QCD SR [9] & 0.75 & 1.69 & 1.69 \\
3PSR [8] & 0.63 & 0.69 & 1.03 \\
QM [52] & 0.68 & 0.66 & 0.96 \\
\hline \hline
\end{tabular}

In agreement with the previous choice of Ref. [9], we take the continuum threshold for the TFFs $A_{1,2}\left(q^{2}\right)$ and $V\left(q^{2}\right)$ as $s_{0}=42.0(5) \mathrm{GeV}^{2}$, which is smaller than the value used for LCSRs under the traditional correlator [51]. This choice in some sense ensures that the contributions from the unwanted scalar resonances that are introduced by using the chiral correlator are greatly suppressed. The Borel windows are determined to be $M^{2}\left(A_{1}\right)=9.8(3)$, $M^{2}\left(A_{2}\right)=11.0(3)$, and $M^{2}(V)=11.0(3)$. We present the TFFs at the maximum recoil point $q^{2}=0$ in Table III, where the errors are squared averages of all the error sources for the LCSRs. As a comparison, we also present the predictions from the NLO $\mathrm{pQCD}$ prediction under PMC scale setting [17], the QCD sum rule with a right-handed correlator [9], the 3PSR (with the Coloumb corrections included) [8], and the QM [52]. It has been pointed out that the LCSRs under various choices of correlators should be consistent with each other under the same input parameters; the $B \rightarrow K^{*}$ TFFs are such examples [53]. Table III shows our LCSR predictions on the TFFs are larger than previous SR predictions, which however agrees with the PMC prediction within errors. The $\mathrm{pQCD}$ prediction is reliable at the maximum recoil point; thus our present LCSR prediction could be treated as a cross-check of the NLO pQCD prediction.

The validity of the LCSR approach is restricted to the kinematical regime of large meson energies, and for the present case, the allowable region for $q^{2}$ is very close to its whole physical region, e.g., $m_{\ell}^{2} \leq q^{2} \leq\left(m_{B_{c}^{+}}-m_{J / \psi}\right)^{2} \approx$ $10 \mathrm{GeV}^{2}$. Thus we do not need to do extra extrapolations for the LCSR TFFs, while the $\mathrm{pQCD}$ prediction is reliable only around the maximum recoil point and a certain modeldependent extrapolation must be made, introducing extra model dependence into the $\mathrm{pQCD}$ prediction. We present the total differential decay width for the $B_{c}^{+} \rightarrow J / \psi \ell^{+} \nu_{\ell}$ $(\ell=e, \mu)$ versus $q^{2}$ by adopting the SVZ sum rules under the BFT (BFTSR) for the twist-2 LCDA in Fig. 3, where the uncertainties are squared averages of the error sources. The PMC prediction with a monopole extrapolation [17] is presented as a comparison. The BFTSR prediction agrees with the PMC prediction in the low and intermediate $q^{2}$

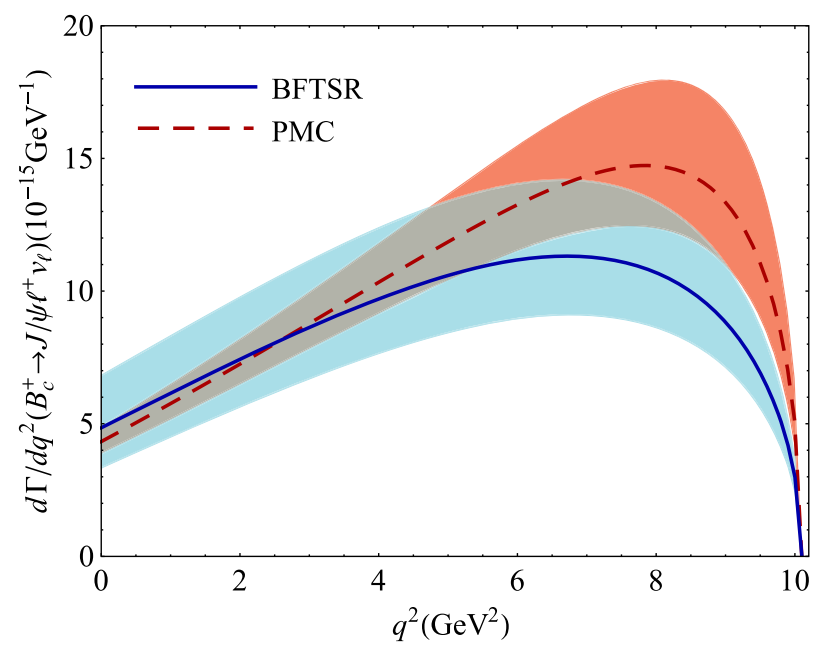

FIG. 3. Differential decay width for the $B_{c}^{+} \rightarrow J / \psi \ell^{+} \nu_{\ell}$ $(\ell=e, \mu)$ versus $q^{2}$ by using the chiral LCSR for the TFFs and by adopting the BFTSR for the twist- 2 LCDA. The PMC prediction [17] is presented as a comparison.

region, but is smaller than the PMC one in the large $q^{2}$ region. This difference leads to a slightly larger integrated decay width for the PMC prediction, but they are consistent with each other within reasonable errors.

After integrating over the allowable $q^{2}$ region, we get the total decay widths for $B_{c}^{+} \rightarrow J / \psi \ell^{+} \nu_{\ell}(\ell=e, \mu)$, which are presented in Table IV. We also present the results from the NLO pQCD prediction under PMC scale setting [17], NLO pQCD prediction under conventional scale setting [12], the QCD sum rules predictions [8,9], LO pQCD prediction [7], QCD relativistic potential model prediction [6], prediction from the Bethe-Salpeter equation [5], and constituent quark model predictions $[3,4]$ in Table IV.

TABLE IV. Total decay width (in units $10^{-15} \mathrm{GeV}$ ) for the $B_{c}^{+} \rightarrow J / \psi \ell^{+} \nu_{\ell}$ by using the chiral LCSR for the TFFs and by adopting the BFTSR for the $J / \psi$ meson twist-2 LCDA. As a comparison, we present the results from the CDF measurement in 2016 [2] and predictions derived under various approaches, i.e., the PMC [17], NLO pQCD calculation [12], QCD sum rules [8,9], LO pQCD calculation [7], QCD relativistic PM [6], BetheSalpeter equation [5], and CQM [3,4].

\begin{tabular}{lc}
\hline \hline References & $\Gamma\left(B_{c}^{+} \rightarrow J / \psi \ell^{+} \nu_{\ell}\right)$ \\
\hline This work & $89.67_{-19.06}^{+24.76}$ \\
PMC [17] & $106.31_{-14.01}^{+18.59}$ \\
NLO pQCD [12] & $97.30_{-20.33}^{+36.22}$ \\
LCSR [9] & $28 \pm 5$ \\
3PSR [8] & 34.69 \\
LO pQCD [7] & $14.7_{-1.73}^{+1.94}$ \\
PM [6] & 30.2 \\
BS equation [5] & 34.4 \\
CQM-I [4] & $21.9^{+1.2}$ \\
CQM-II [3] & 28.2 \\
\hline \hline
\end{tabular}


Being consistent with Table III, our prediction of $\Gamma\left(B_{c}^{+} \rightarrow J / \psi \ell^{+} \nu_{\ell}\right)=89.67_{-19.06}^{+24.76} \times 10^{-15} \mathrm{GeV}$ is about three times larger than previous SR predictions, but agrees with the extrapolated NLO pQCD predictions within errors. It is found that a larger decay width is helpful for explaining the large value of $\Re\left(J / \psi \ell^{+} \nu_{\ell}\right)$ derived from the CDF Run II data [2]. Thus we think a reliable prediction on the semileptonic decay $B_{c}^{+} \rightarrow J / \psi \ell^{+} \nu_{\ell}$ can be achieved by applying the sum rules approach.

\section{A discussion on the ratio $\Re\left(J / \psi \ell^{+} \nu_{\ell}\right)$}

Taking the hadronization fractions $f_{b \rightarrow B_{c}^{+}}=(1.3 \pm$ $0.2) \times 10^{-3}, \quad f_{\bar{b} \rightarrow B^{+}}=0.404 \pm 0.006, \quad$ and $\mathcal{B}\left(B^{+} \rightarrow\right.$ $\left.J / \psi K^{+}\right)=(1.026 \pm 0.031) \times 10^{-3}$ [41], we obtain the value of $\Re\left(J / \psi \ell^{+} \nu_{\ell}\right)$ defined in Eq. (1). The value of $\Re\left(J / \psi \ell^{+} \nu_{\ell}\right)$ as a function of $B_{c}^{+}$meson lifetime $\tau_{B_{c}^{+}}$is presented in Fig. 4. The CDF measurements [1,2,54-56] as shown in Table $\mathrm{V}$ have also been presented in Fig. 4, where all the errors are added in quadrature. Theoretical predictions on $\Re\left(J / \psi \ell^{+} \nu_{\ell}\right)$ are close in shape, all of which increase with the increment of $\tau_{B_{c}^{+}}$. In comparison to previous LCSR prediction such as that of Ref. [8] and the LO pQCD prediction [7], our prediction of $\Re\left(J / \psi \ell^{+} \nu_{\ell}\right)$ shows a better agreement with the CDF measurements, which is also consistent with the PMC NLO pQCD prediction [17].

If setting the $B_{c}^{+}$meson lifetime as the PDG averaged value, $\tau_{B_{c}^{+}}=0.507 \pm 0.009 \mathrm{ps}$ [41], we get the value of $\Re\left(J / \psi \ell^{+} \nu_{\ell}\right)$, which is listed in Table $\mathrm{V}$, in which the predictions by using the total decay width $\Gamma\left(B_{c}^{+} \rightarrow\right.$ $\left.J / \psi \ell^{+} \nu_{\ell}\right)$ of Refs. [3-9,12,17] are also listed. Table V

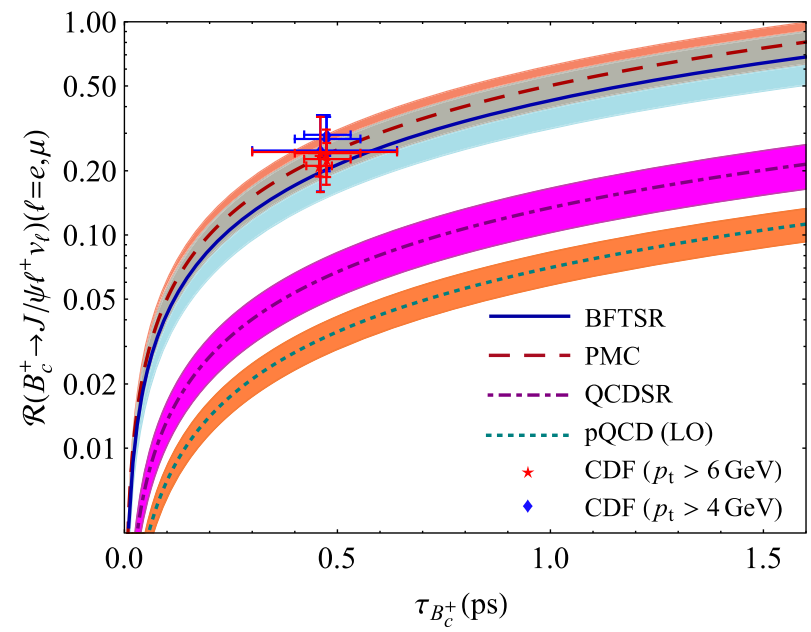

FIG. 4. The value of $\Re\left(J / \psi \ell^{+} \nu_{\ell}\right)$ versus the $B_{c}^{+}$meson lifetime $\tau_{B_{c}^{+}}$by using the chiral LCSR for the TFFs and by adopting the BFTSR for the twist-2 LCDA. The PMC [17], QCD SR prediction [8], and LO $\mathrm{pQCD}$ prediction [7] are presented as a comparison. The lines are their central values and the shaded bands are their errors. The CDF measurements [1,2,54-56] are also presented.
TABLE V. Our prediction of $\sigma \cdot \mathcal{B}$ ratio $\Re\left(J / \psi \ell^{+} \nu_{\ell}\right)$. Various theoretical predictions are presented as a comparison. The CDF measurement in 2016 [2] is also presented, where the symbols "st" and "sy" stand for the statistical error and the systematic error, respectively.

\begin{tabular}{lc}
\hline \hline References & $\Re\left(J / \psi \ell^{+} \nu_{\ell}\right)$ \\
\hline This work & $0.217_{-0.057}^{+0.069}$ \\
CDF2016 [2] & $0.211 \pm 0.012(\mathrm{st})_{-0.020}^{+0.021}(\mathrm{sy})$ \\
PMC [17] & $0.257_{-0.034}^{+0.045}$ \\
NLO pQCD [12] & $0.235_{-0.049}^{+0.088}$ \\
QCDSR-LCSR [9] & $0.068(12)$ \\
QCDSR-3PSR [8] & 0.084 \\
LO pQCD [7] & $0.036_{-0.004}^{+0.005}$ \\
PM [6] & 0.073 \\
BS equation [5] & 0.083 \\
CQM-I [4] & $0.053^{+0.003}$ \\
CQM-II [3] & 0.068 \\
\hline \hline
\end{tabular}

shows that our prediction agrees with the data issued by the CDF Collaboration in 2016 [2].

\section{SUMMARY}

The LCDA is an important component for QCD exclusive processes. In the paper, we make a detailed study on the $J / \psi$ longitudinal leading-twist LCDA $\phi_{2 ; J / \psi}^{\|}$by using the QCD sum rules within the BFT. The moments of the LCDA $\phi_{2 ; J / \psi}^{\|}$are presented in Table I, in which the contributions from the LO terms, NLO terms, dimensionfour operators, and dimension-six operators are presented separately. It shows that the LO terms are dominant, which provide $\sim 95 \%$ contribution to $\left\langle\xi_{2 ; J / \psi}\right\rangle, \sim 91 \%$ to $\left\langle\xi_{4 ; J / \psi}\right\rangle$, and $\sim 94 \%$ to $\left\langle\xi_{6 ; J / \psi}\right\rangle$, respectively. The contributions of the dimension-four and dimension-six condensates are small, and their contributions do not follow the power counting of $1 / M^{2}$ suppression. Thus the contribution from the dimension-six condensate has the same importance as that of the dimension-four condensate, which is also helpful for determining more precise input parameters for the SRs. By further using the relations (15), we obtain the first three Gegenbauer moments at scale $\mu_{c}=\bar{m}_{c}\left(\bar{m}_{c}\right)$, $a_{2 ; J / \psi}^{\|}\left(\mu_{c}\right)=-0.340(34), \quad a_{4 ; J / \psi}^{\|}\left(\mu_{c}\right)=0.071(28), \quad$ and $a_{6 ; J / \psi}^{\|}\left(\mu_{c}\right)=0.002(1)$.

As an application of the derived $\phi_{2 ; J / \psi}^{\|}$, we have studied the $B_{c}$ meson semileptonic decay $B_{c}^{+} \rightarrow J / \psi \ell^{+} \nu_{\ell}$, which is one of the golden channels for observing the $B_{c}$ meson. Table III shows that our LCSR predictions on the TFFs are larger than previous SR predictions, but agree with the extrapolated NLO pQCD prediction within errors. This leads to a larger prediction of the decay width, $\Gamma\left(B_{c}^{+} \rightarrow\right.$ $\left.J / \psi \ell^{+} \nu_{\ell}\right)=89.67_{-19.06}^{+24.76} \times 10^{-15} \mathrm{GeV}$, which explains the large value of $\Re\left(J / \psi \ell^{+} \nu_{\ell}\right)$ obtained by the CDF Run II 
data [2], as shown by Fig. 4. More explicitly, if setting $\tau_{B_{c}^{+}}=0.507 \pm 0.009$ ps [41], we obtain $\Re\left(J / \psi \ell^{+} \nu_{\ell}\right)=$ $0.217_{-0.057}^{+0.069}$, which agrees well with the CDF predictions in 2016 and the PMC NLO pQCD prediction.

\section{ACKNOWLEDGMENTS}

We are grateful to Jian-Ming Shen for helpful discussions. This work was supported in part by the Natural
Science Foundation of China under Grants No. 11647112, No. 11625520, No. 11547015, and No. 11765007; the Project of Guizhou Provincial Department of Science and Technology under Grant No. [2017]1089; the Project for Young Talents Growth of Guizhou Provincial Department of Education under Grant No. KY[2016]156; and the Key Project for Innovation Research Groups of Guizhou Provincial Department of Education under Grant No. KY[2016]028.
[1] F. Abe et al. (CDF Collaboration), Observation of the $B_{c}$ Meson in $p \bar{p}$ Collisions at $\sqrt{s}=1.8 \mathrm{TeV}$, Phys. Rev. Lett. 81, 2432 (1998).

[2] T. A. Aaltonen et al. (CDF Collaboration), Measurement of the $B_{c}^{ \pm}$production cross section in $p \bar{p}$ collisions at $\sqrt{s}=1.96$ TeV, Phys. Rev. D 93, 052001 (2016).

[3] M. A. Ivanov, J. G. Korner, and P. Santorelli, Semileptonic decays of $B_{c}$ mesons into charmonium states in a relativistic quark model, Phys. Rev. D 71, 094006 (2005).

[4] E. Hernandez, J. Nieves, and J. M. Verde-Velasco, Study of exclusive semileptonic and nonleptonic decays of $B_{c}$-in a nonrelativistic quark model, Phys. Rev. D 74, 074008 (2006).

[5] C. H. Chang, Y. Q. Chen, G. L. Wang, and H. S. Zong, Decays of the meson $B_{c}$ to a $\mathrm{P}$ wave charmonium state $\chi_{c}$ or $h_{c}$, Phys. Rev. D 65, 014017 (2001).

[6] A. Abd El-Hady, J. H. Munoz, and J. P. Vary, Semileptonic and nonleptonic $B_{c}$ decays, Phys. Rev. D 62, 014019 (2000).

[7] W. F. Wang, Y. Y. Fan, and Z. J. Xiao, Semileptonic decays $B_{c} \rightarrow\left(\eta_{c}, J / \Psi\right) l \nu$ in the perturbative QCD approach, Chin. Phys. C 37, 093102 (2013).

[8] V. V. Kiselev, A. K. Likhoded, and A. I. Onishchenko, Semileptonic $B_{c}$ meson decays in sum rules of QCD and NRQCD, Nucl. Phys. B569, 473 (2000).

[9] T. Huang and F. Zuo, Semileptonic $B_{c}$ decays and charmonium distribution amplitude, Eur. Phys. J. C 51, 833 (2007).

[10] I. I. Balitsky, V. M. Braun, and A. V. Kolesnichenko, Radiative decay $\Sigma^{+} \rightarrow p \gamma$ in quantum chromodynamics, Nucl. Phys. B312, 509 (1989).

[11] V. L. Chernyak and I. R. Zhitnitsky, $B$ meson exclusive decays into baryons, Nucl. Phys. B345, 137 (1990).

[12] C. F. Qiao and R. L. Zhu, Estimation of semileptonic decays of $B_{c}$ meson to $\mathrm{S}$-wave charmonia with nonrelativistic QCD, Phys. Rev. D 87, 014009 (2013).

[13] S. J. Brodsky and X.G. Wu, Scale setting using the extended renormalization group and the principle of maximum conformality: The QCD coupling constant at four loops, Phys. Rev. D 85, 034038 (2012).

[14] S. J. Brodsky and X. G. Wu, Eliminating the Renormalization Scale Ambiguity for Top-Pair Production Using the Principle of Maximum Conformality, Phys. Rev. Lett. 109, 042002 (2012).
[15] M. Mojaza, S. J. Brodsky, and X. G. Wu, Systematic AllOrders Method to Eliminate Renormalization-Scale and Scheme Ambiguities in Perturbative QCD, Phys. Rev. Lett. 110, 192001 (2013).

[16] S. J. Brodsky, M. Mojaza, and X. G. Wu, Systematic scale setting to all orders: The principle of maximum conformality and commensurate scale relations, Phys. Rev. D 89, 014027 (2014).

[17] J. M. Shen, X. G. Wu, H. H. Ma, and S. Q. Wang, QCD corrections to the $B_{c}$ to charmonia semileptonic decays, Phys. Rev. D 90, 034025 (2014).

[18] P. Ball and R. Zwicky, $B_{d, s} \rightarrow \rho, \omega, K^{*}, \phi$ decay form factors from light-cone sum rules revisited, Phys. Rev. D 71, 014029 (2005).

[19] P. Ball, V. M. Braun, Y. Koike, and K. Tanaka, Higher twist distribution amplitudes of vector mesons in QCD: Formalism and twist-three distributions, Nucl. Phys. B529, 323 (1998).

[20] P. Ball and V. M. Braun, Higher twist distribution amplitudes of vector mesons in QCD: Twist-4 distributions and meson mass corrections, Nucl. Phys. B543, 201 (1999).

[21] T. Huang, Z. H. Li, and X. Y. Wu, Improved approach to the heavy to light form factors in the light-cone QCD sum rules, Phys. Rev. D 63, 094001 (2001).

[22] Z. G. Wang, M. Z. Zhou, and T. Huang, $B \rightarrow \pi$ weak form factor with chiral current in the light-cone sum rules, Phys. Rev. D 67, 094006 (2003).

[23] X. G. Wu, T. Huang, and Z. Y. Fang, SU SU (3)-symmetry breaking effects of the $B \rightarrow K$ transition form factor in the QCD light-cone sum rules, Phys. Rev. D 77, 074001 (2008).

[24] H. B. Fu, X. G. Wu, H. Y. Han, Y. Ma, and H. Y. Bi, The $\rho$ meson longitudinal leading-twist distribution amplitude, Phys. Lett. B 738, 228 (2014).

[25] H. B. Fu, X. G. Wu, W. Cheng, and T. Zhong, $\rho$-meson longitudinal leading-twist distribution amplitude within QCD background field theory, Phys. Rev. D 94, 074004 (2016).

[26] P. Ball and V. M. Braun, Use and misuse of QCD sum rules in heavy to light transitions: The decay $B \rightarrow \rho e \nu_{e}$ reexamined, Phys. Rev. D 55, 5561 (1997).

[27] A. E. Bondar and V. L. Chernyak, Is the BELLE result for the cross section $\sigma\left(e^{+} e^{-} \rightarrow J / \psi+\eta_{c}\right)$ a real difficulty for QCD? Phys. Lett. B 612, 215 (2005). 
[28] G. T. Bodwin, D. Kang, and J. Lee, Reconciling the light-cone and NRQCD approaches to calculating $e^{+} e^{-} \rightarrow$ $J / \psi+\eta_{c}$, Phys. Rev. D 74, 114028 (2006).

[29] Y. J. Sun, X. G. Wu, F. Zuo, and T. Huang, The cross section of the process $\mathrm{e}^{+}+\mathrm{e}^{-} \rightarrow \mathrm{J} / \psi+\eta(\mathrm{c})$ within the QCD lightcone sum rules, Eur. Phys. J. C 67, 117 (2010).

[30] V. L. Chernyak and A. R. Zhitnitsky, Asymptotic behavior of exclusive processes in QCD, Phys. Rep. 112, 173 (1984).

[31] G. P. Lepage and S. J. Brodsky, Exclusive processes in perturbative quantum chromodynamics, Phys. Rev. D 22, 2157 (1980).

[32] M. A. Shifman, A. I. Vainshtein, and V. I. Zakharov, QCD and resonance physics. Theoretical foundations, Nucl. Phys. B147, 385 (1979).

[33] J. Govaerts, F. de Viron, D. Gusbin, and J. Weyers, Exotic mesons from QCD sum rules, Phys. Lett. 128B, 262 (1983).

[34] T. Huang and Z. Huang, Quantum chromodynamics in background fields, Phys. Rev. D 39, 1213 (1989).

[35] T. Zhong, X. G. Wu, Z. G. Wang, T. Huang, H. B. Fu, and H. Y. Han, Revisiting the pion leading-twist distribution amplitude within the QCD background field theory, Phys. Rev. D 90, 016004 (2014).

[36] V. A. Novikov, M. A. Shifman, A. I. Vainshtein, and V. I. Zakharov, Calculations in external fields in quantum chromodynamics. Technical Review, Fortschr. Phys. 32, 585 (1984).

[37] W. Hubschmid and S. Mallik, Operator expansion at short distance in QCD, Nucl. Phys. B207, 29 (1982).

[38] M. A. Shifman, Wilson loop in vacuum fields, Nucl. Phys. B173, 13 (1980).

[39] P. Colangelo and A. Khodjamirian, in QCD sum rules, a modern perspective, edited by M. Shifman, At the Frontier of Particle Physics Vol. 3 (World Scientific, Singapore, 2001), pp. 1495-1576.

[40] P. Ball and V. M. Braun, The Rho meson light-cone distribution amplitudes of leading-twist revisited, Phys. Rev. D 54, 2182 (1996).
[41] C. Patrignani et al. (Particle Data Group), Review of particle physics, Chin. Phys. C 40, 100001 (2016).

[42] D. S. Hwang and G. H. Kim, Decay constant ratios $f_{\eta_{c}} / f_{J / \psi}$ and $f_{\eta_{b}} / f_{\Upsilon}$, Z. Phys. C 76, 107 (1997).

[43] V. V. Braguta, A. K. Likhoded, and A. V. Luchinsky, The study of leading-twist light-cone wave function of $\eta_{c}$ meson, Phys. Lett. B 646, 80 (2007).

[44] E. G. Floratos, D. A. Ross, and C. T. Sachrajda, Higher order effects in asymptotically free gauge theories: The anomalous dimensions of Wilson operators, Nucl. Phys. B 129, 66 (1977).

[45] D. Mueller, The evolution of the pion distribution amplitude in next-to-leading-order, Phys. Rev. D 51, 3855 (1995).

[46] P. Ball and R. Zwicky, $\left|V_{t d} / V_{t s}\right|$ from $B \rightarrow V \gamma$, J. High Energy Phys. 04 (2006) 046.

[47] V. V. Braguta, The study of leading-twist light-cone wave functions of $J / \psi$ meson, Phys. Rev. D 75, 094016 (2007).

[48] W. Buchmuller and S. H. H. Tye, Quarkonia and quantum chromodynamics, Phys. Rev. D 24, 132 (1981).

[49] E. Eichten, K. Gottfried, T. Kinoshita, K. D. Lane, and T. M. Yan, Charmonium: The model, Phys. Rev. D 17, 3090 (1978); Erratum, Phys. Rev. D21, 313(E) (1980).

[50] G. T. Bodwin, D. Kang, and J. Lee, Potential-model calculation of an order-v(2) NRQCD matrix element, Phys. Rev. D 74, 014014 (2006).

[51] M. Chabab, On the determination of the leptonic decay constant $f_{B_{c}}$ from QCD sum rules, Phys. Lett. B 325, 205 (1994).

[52] M. A. Ivanov, J. G. Korner, and P. Santorelli, The semileptonic decays of the $B_{c}$ meson, Phys. Rev. D 63, 074010 (2001).

[53] W. Cheng, X. G. Wu, and H. B. Fu, Reconsideration of the $B \rightarrow K^{*}$ transition form factors within the QCD light-cone sum rules, Phys. Rev. D 95, 094023 (2017).

[54] CDF Collaboration, CDF Report No. 7649, 2005.

[55] CDF Collaboration, CDF Report No. 7926, 2005.

[56] CDF Collaboration, CDF Report No. 9740-CONF, 2009. 